\title{
Hydroxychloroquine-induced retinopathy in a 57-year-old woman
}

\author{
Alex Ragan MD, Michael Fielden MD
}

Cite as: CMAJ 2017 January 16;189:E69-72. doi: 10.1503/cmaj.150716

A

57-year-old woman was referred to a retinal specialist with concerns of ocular toxicity from hydroxychloroquine. She complained of blurry vision and flashing lights (photopsia). The patient had been taking $400 \mathrm{mg}$ of hydroxychloroquine daily for 10 years for the management of systemic lupus erythematosus.

At presentation, the calculated dose of hydroxychloroquine was $5.3 \mathrm{mg} / \mathrm{kg}$ (based on a daily dose of $400 \mathrm{mg}$ and the patient's weight of $75 \mathrm{~kg}$ ); however, because the patient had progressively gained weight over the course of her treatment, the average dose per kilogram over the treatment period was higher. The cumulative dose at the time of presentation was estimated to be about $1460 \mathrm{~g}$.

The patient's medical history was otherwise unremarkable. Specifically, she had no kidney or liver dysfunction and no previous ocular problems, including macular disorders. She was taking no other medications.

The patient's visual acuity at presentation was 20/20 (right eye) and 20/25 (left eye). Colour vision was notably impaired: the patient could correctly read only 8 of 16 Ishihara colour vision plates with the right eye and 1 of 16 plates with the left eye (normal 16/16). The only abnormality on ocular examination was subtle macular depigmentation in a bull's-eye configuration bilaterally (Figure 1).

\section{KEY POINTS}

- Retinal toxicity from long-term hydroxychloroquine use is common, occurring in as many as $7.5 \%$ of patients.

- Retinal damage from hydroxychloroquine use has no known treatment and is largely irreversible.

- Patients starting a long-term course of hydroxychloroquine treatment should be referred to an ophthalmologist for baseline retinal testing.

- For patients with a normal risk profile, annual screening for hydroxychloroquine-induced retinopathy should begin five years after the start of treatment.

- For patients with major risk factors (e.g., high cumulative or daily dose, duration of treatment longer than five years, and kidney or liver disease), annual screening should begin one year after the start of treatment.

Automated visual field testing, spectral domain optical coherence tomography (OCT), fundus autofluorescence and fluorescein angiography were performed to elucidate further the nature and cause of the retinal pathology. Visual field testing revealed a

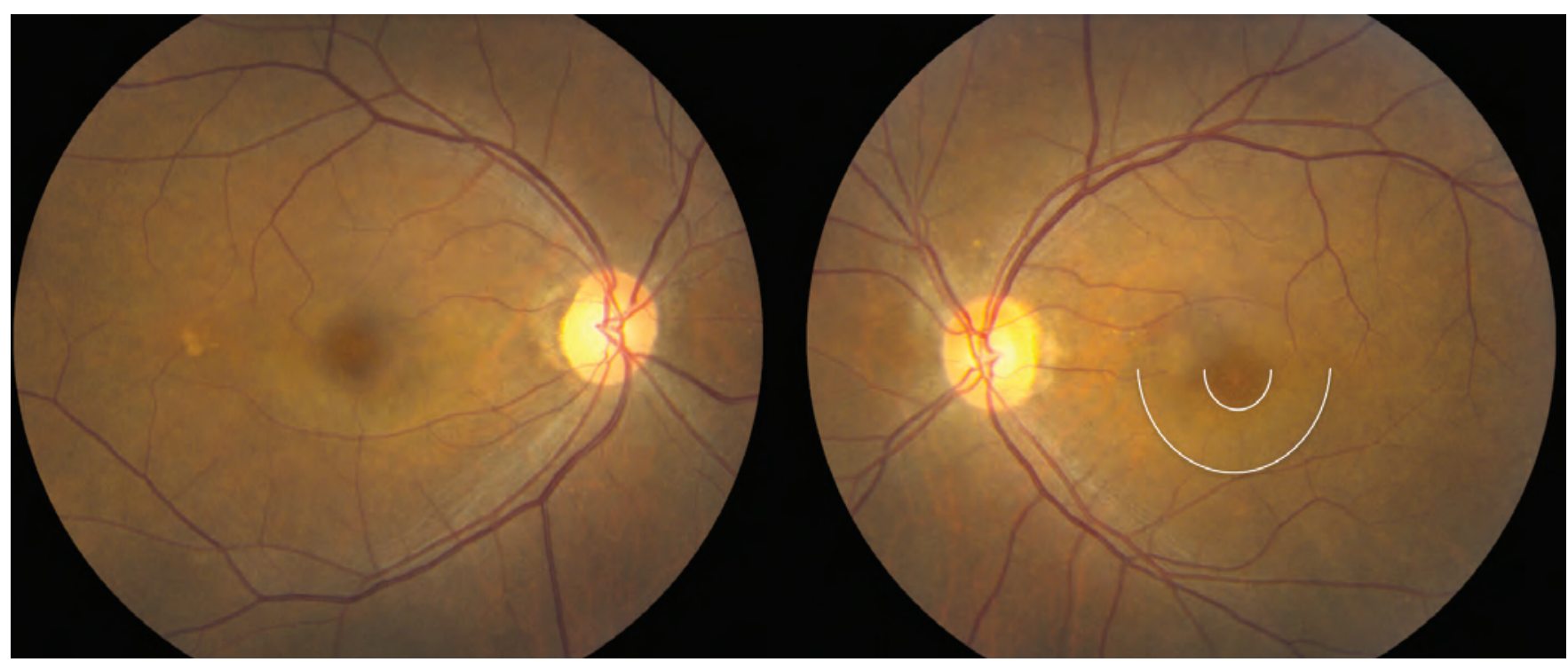

Figure 1: Funduscopic examination showing subtle paracentral depigmentation of the retinal pigment epithelium sparing the central fovea (bull's-eye maculopathy) in the right and left eyes of a 57-year-old woman 10 years after the start of hydroxychloroquine treatment. The two arcs on the photograph of the left eye highlight the inner and outer margins of the inferior aspect of the bull's eye, which encircles the fovea. The same pattern of paracentral depigmentation is seen in the right eye. 
dense annular visual field defect in each eye (Figure 2). Spectral domain OCT showed substantial parafoveal outer retinal atrophy in both eyes (Figure 3). Fundus autofluorescence showed areas of hyper- and hypoautofluorescence (Appendix 1, available at www. cmaj.ca/lookup/suppl/doi:10.1503/cmaj.150716/-/DC1), which correlated with the changes observed on visual field and OCT testing.

Bilateral retinopathy secondary to the use of hydroxychloroquine was diagnosed. The patient was advised to refrain from using hydroxychloroquine and chloroquine in perpetuity, and her rheumatologist and family doctor were informed of the same.

The patient was followed over the next several years. Her visual acuity declined further, to $20 / 30$ in the right eye and 20/50 in the left eye, and visual field testing showed progressive deterioration (Figure 2).
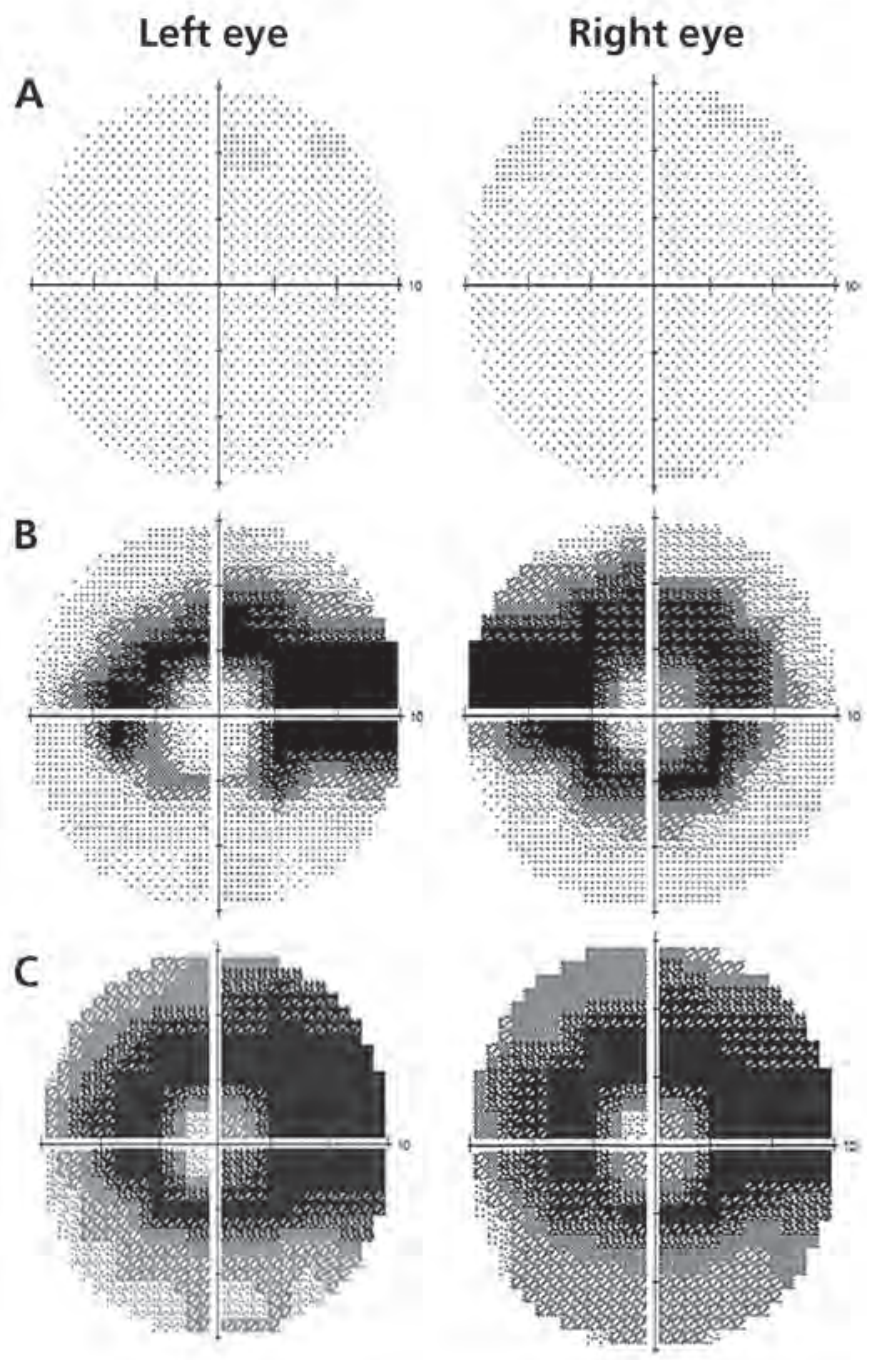

Figure 2: Images from 10-2 visual field testing using a Humphrey field analyzer, showing (A) normal findings from a control subject for comparative purposes; (B) a dense annular visual field defect in each eye of the patient at presentation; and $(C)$ progression of the visual field defects about two years after the cessation of hydroxychloroquine treatment.

\section{Discussion}

Hydroxychloroquine is commonly used to treat systemic lupus erythematosus, rheumatoid arthritis, dermatomyositis, Sjögren syndrome, and other autoimmune and connective tissue disorders. ${ }^{1}$ Despite the drug's effectiveness, long-term use increases the risk of retinopathy. ${ }^{2}$

Short-term use of hydroxychloroquine (less than five years) does not typically lead to permanent adverse retinal effects. ${ }^{3}$ However, long-term use has an alarmingly high incidence of toxicity: a recent study reported an overall prevalence with long-term use of $7.5 \%{ }^{4}$

Not only is hydroxychloroquine-induced retinopathy more common than previously thought, ${ }^{4}$ it is pernicious in its onset and largely irreversible. Moreover, the toxic effects have been shown to progress even after treatment has stopped. ${ }^{5}$

Chloroquine, a predecessor and chemical analog to hydroxychloroquine, is indicated for the treatment of many of the same diseases as its successor. The retinotoxic effects of this drug are similar but more pronounced than those attributable to hydroxychloroquine. ${ }^{6}$ Accordingly, the use of chloroquine has fallen out of favour.

\section{Development of retinopathy}

Although initially asymptomatic, hydroxychloroquine-induced retinopathy develops slowly, producing paracentral scotomas (blind spots near the central vision) that patients may begin to notice while reading. ${ }^{7,8}$ As the retinopathy worsens, central visual acuity and paracentral vision are increasingly affected. Patients may also complain of glare, blurred vision, photopsia and metamorphopsia (visual distortions). ${ }^{8}$ On funduscopic examination, characteristic depigmentation of the parafoveal retinal pigment epithelium will slowly emerge, with the classic bull's-eye maculopathy (a retinopathy specific to the geographic area of the macula) appearing in the late stages of toxicity, once permanent damage has occurred. ${ }^{7,8}$

Although there is some evidence that cessation of treatment in cases of early retinopathy may result in reversal of toxic damage and functional visual loss, ${ }^{5}$ moderate to severe retinopathy is permanent. ${ }^{4}$ This underscores the importance of screening to detect early signs of toxicity and prevent more serious retinal damage.

\section{Risk factors}

Major risk factors for hydroxychloroquine-induced retinopathy include a cumulative dose of more than $1000 \mathrm{~g},{ }^{3}$ a daily dose of more than $6.5 \mathrm{mg} / \mathrm{kg}$, ${ }^{3}$ duration of treatment longer than five years, ${ }^{9} \mathrm{de}-$ velopment of age-related changes to the eye, ${ }^{10}$ pre-existing retinopathy, ${ }^{7}$ kidney or liver disease,${ }^{4}$ and concurrent use of tamoxifen. ${ }^{4}$

Although our patient was taking what is considered to be a safe daily dose at her current weight $(5.3 \mathrm{mg} / \mathrm{kg})$, toxic retinopathy nevertheless developed. Even if the daily dose is deemed safe, other risk factors such as cumulative dose and duration of therapy must be taken into account as part of routine surveillance.

\section{Screening}

Given the frequency, severity and irreversibility of hydroxychloroquine-induced retinopathy, it is incumbent upon physicians to identify and screen patients at risk. The American Academy of 
Ophthalmology has issued revised guidelines for the screening of patients taking long-term hydroxychloroquine therapy. ${ }^{7}$

All patients prescribed long-term hydroxychloroquine therapy should have a baseline examination conducted by an ophthalmologist at the start of treatment. The purposes of the examination are to identify pre-existing maculopathies that may obscure later changes due to hydroxychloroquine, to obtain baseline results from ocular testing (OCT and visual field testing) and to stratify patients' risk for the purpose of follow-up. ${ }^{7}$

Patients who have a normal risk profile should undergo annual screening by an ophthalmologist beginning five years after the start of hydroxychloroquine treatment. For those who have major risk factors (e.g., high cumulative or daily dose, duration of treatment longer than five years and kidney or liver disease), annual screening should begin one year after the start of treatment and may be conducted more frequently (i.e., biannually) depending on their risk factors. Regardless of risk profile, screening should occur more frequently as the duration of treatment, and the risk of toxicity, increases. ${ }^{7}$

The screening performed by the ophthalmologist should include a thorough ophthalmic examination to detect macular pigmentary changes. ${ }^{7}$ Visual field testing should also be performed with 10-2 perimetry using a Humphrey field analyzer. In addition, at least one of the following objective tests should be administered: spectral domain OCT, multifocal electroretinography or fundus autofluorescence. ${ }^{7}$

Spectral domain OCT shows a cross-section of the retina (Figure 3) and, in patients with hydroxychloroquine-induced retinopathy, may show findings such as loss of foveal depression, perifoveal loss of the retinal ellipsoid zone causing a "saucer sign," posterior displacement of the inner retinal structures toward the retinal pigment epithelium (the "sink-hole effect") and thinning of the outer nuclear layer in a perifoveal distribution (Figure 3). Multifocal electroretinography records bioelectrical potential in the retina upon stimulation
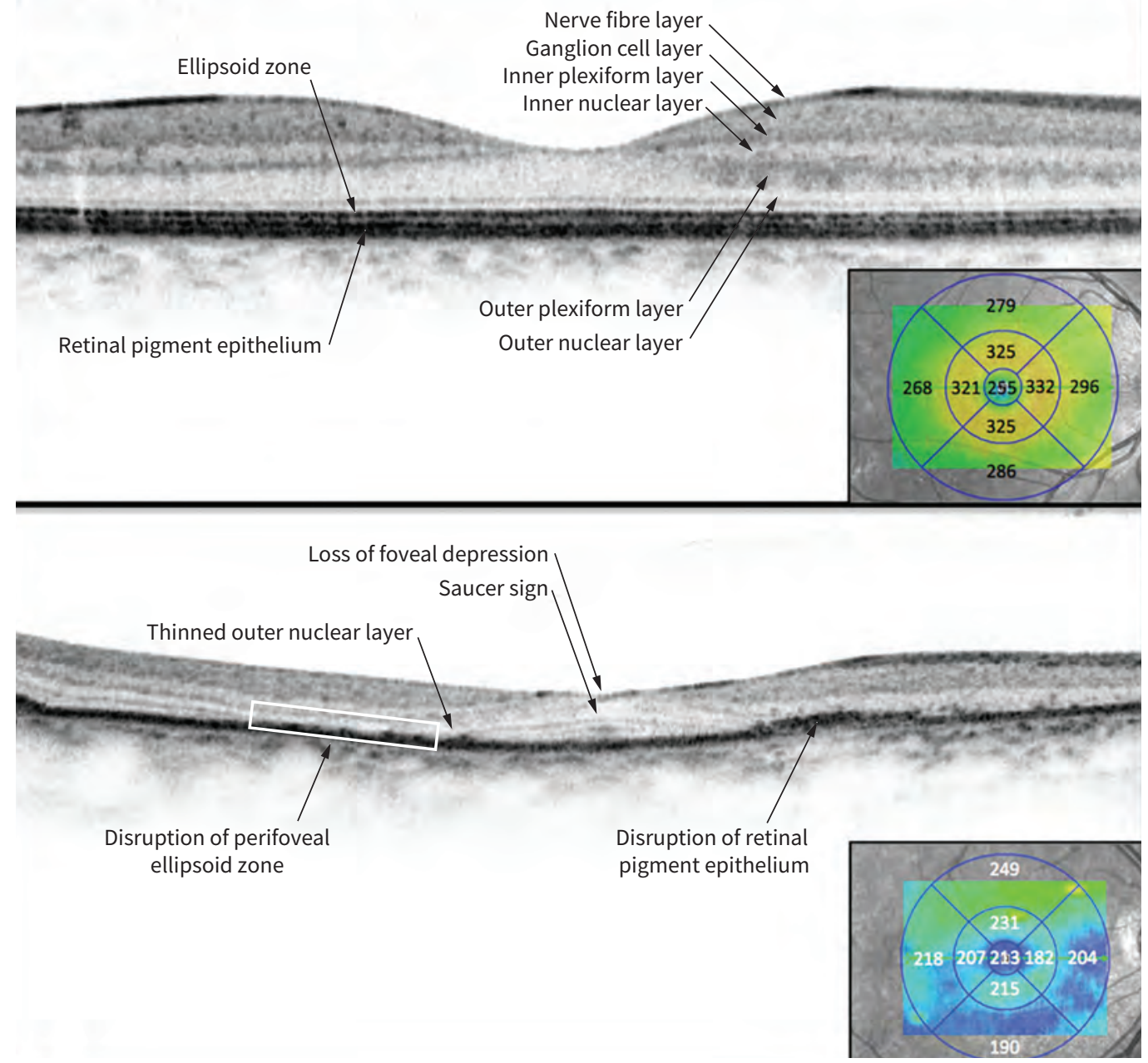

Figure 3: Top panel: Spectral domain optical coherence tomography (OCT) image of the right eye of a control subject for comparative purposes; the image represents a horizontal cross-section of the retina across the fovea. Bottom panel: Spectral domain OCT image of the right eye of the patient at the same anatomic position as above, showing substantial loss of the normal foveal depression, thinning and loss of the outer nuclear layer (perifoveal photoreceptors), disruption and irregularity of the retinal pigment epithelium and perifoveal loss of the retinal ellipsoid zone causing a "saucer sign." Insets are retinal thickness maps (measurements in micrometres) showing normal thickness of the control subject's retina (top panel) and substantial parafoveal thinning of the patient's retina (bottom panel). 
with light and is very sensitive to photoreceptor dysfunction; hydroxychloroquine-induced retinopathy will manifest as signal depression in a parafoveal distribution. Fundus autofluorescence images capture naturally fluorescing molecules within the photoreceptor layer and retinal pigment epithelium; both hypo- and hyperfluorescent areas of the retina suggest toxicity (Appendix 1).

Neither the Amsler grid nor visual acuity testing is a reliable screening test for hydroxychloroquine-induced retinopathy. The Amsler grid is too reliant on patient understanding and alertness to be consistent for screening purposes. ${ }^{7}$ Visual acuity testing often yields normal findings until very advanced stages of retinopathy and thus is not useful in screening for early signs of hydroxychloroquine-induced toxicity. Colour vision testing, usually performed with Ishihara plates, may give abnormal results in the early stages of toxicity, but its diagnostic value in this context remains the subject of debate. ${ }^{7}$ Accordingly, colour vision testing should be considered a supplement rather than a replacement to the other recommended testing modalities.

\section{Management}

There is no known treatment of hydroxychloroquine-induced retinopathy. ${ }^{8}$ Management is aimed at preventing further retinal damage. Although some studies suggest that certain retinopathic damage may reverse after treatment is stopped, ${ }^{6}$ true retinopathy, characterized in part by the presence of visual symptoms, is predominantly irreversible. ${ }^{8}$ Accordingly, once toxicity is suspected, timely consideration must be given to the risks and benefits of continuing the use of hydroxychloroquine, reducing the dose or stopping the use of the drug. Consultation with the patient and his or her rheumatologist or primary care physician is necessary to find the optimal therapeutic balance.

In more severe cases of toxicity, characterized by bull's-eye maculopathy and visual field loss, stopping the use of hydroxychloroquine treatment is recommended, unless the benefits to the patient of continued use clearly outweigh the risk of losing central vision. ${ }^{7}$

\section{Counselling patients}

Patients who are starting a long-term course of hydroxychloroquine treatment must be counselled by their rheumatologist or primary care physician regarding both the risk of retinal toxicity and the importance of ophthalmic screening to detect early signs of toxicity. Not only is such counselling necessary to obtain fully informed consent to the treatment, it is crucial if patients are to be sensitive to small changes in their vision. In addition, patients who understand the risks of treatment are less likely to be lost to ophthalmologic follow-up and more likely to have early signs of toxicity detected. ${ }^{7}$

\section{References}

1. Rynes RI. Antimalarial drugs in the treatment of rheumatological diseases. $\mathrm{Br} \mathrm{J}$ Rheumatol 1997;36:799-805.

2. Michaelides M, Stover NB, Francis PJ, et al. Retinal toxicity associated with hydroxychloroquine and chloroquine: risk factors, screening, and progression despite cessation of therapy. Arch Ophthalmol 2011;129:30-9.

3. Mavrikakis I, Sfikakis PP, Mavrikakis E, et al. The incidence of irreversible retinal toxicity in patients treated with hydroxychloroquine: a reappraisal. Ophthalmology 2003;110:1321-6.

4. Melles RB, Marmor MF. The risk of toxic retinopathy in patients on long-term hydroxychloroquine therapy. JAMA Ophthalmol 2014;132:1453-60.

5. Mititelu M, Wong BJ, Brenner M, et al. Progression of hydroxychloroquine toxic effects after drug therapy cessation: new evidence from multimodal imaging. JAMA Ophthalmol 2013;131:1187-97.

6. Rynes RI. Ophthalmologic safety of long-term hydroxychloroquine sulfate treatment. Am J Med 1983;75:35-9.

7. Marmor MF, Kellner U, Lai TYY, et al. Revised recommendations on screening for chloroquine and hydroxychloroquine retinopathy. Ophthalmology 2011; 118:415-22.

8. Yam JCS, Kwok AKH. Ocular toxicity of hydroxychloroquine. Hong Kong Med J 2006; 12:294-304.

9. Wolfe F, Marmor MF. Rates and predictors of hydroxychloroquine retinal toxicity in patients with rheumatoid arthritis and systemic lupus erythematosis. Arthritis Care Res (Hoboken) 2010;94:1637-42.

10. Bergholz R, Schroeter J, Ruther K. Evaluation of risk factors for retinal damage due to chloroquine and hydroxychloroquine. Br J Ophthalmol 2010;94:1637-42.

\section{Competing interests: None declared.}

This article has been peer reviewed.

The authors have obtained patient consent.

Affiliation: Department of Surgery, Cumming School of Medicine, University of Calgary, Calgary, Alta.

Contributors: Michael Fielden was responsible for conception of the project and the critical revision of drafts for intellectual content. Alex Ragan was responsible for the research, analysis, drafting and revision of the manuscript. Both of the authors approved the version to be published and agreed to act as guarantors of the work.

Correspondence to: Alex Ragan, amragan@ucalgary.ca

The section Cases presents brief case reports that convey clear practical lessons. Preference is given to common presentations of important rare conditions, and important unusual presentations of common problems. Articles start with a case presentation (500 words maximum), and a discussion of the underlying condition follows (1000 words maximum). Visual elements (e.g., tables of the differential diagnosis, clinical features or diagnostic approach) are encouraged. Consent from patients for publication of their story is a necessity. See information for authors at www.cmaj.ca. 